\title{
Connection between Parameters of Timing Diagram of Automatic Machine and Equations Movements of Mechanisms
}

\author{
A. Jomartov, S. Joldasbekov, and Y. Temirbekov, Member, IACSIT
}

\begin{abstract}
Mechanical design engineers who have to make a machine or modify the machine usually deal with designing timing diagram of related movements between each part in the machine. Timing diagram is a useful tool for the designer not only to see how each part of the machine works together, but also to see the opportunity to improve the machine movement through "overlap" motion. The aim of this paper is developed a vector model of timing diagram automatic machine, which allows us to solve a variety of dynamic tasks by changing the parameters timing diagram of its mechanisms. There is used the method presentation linear timing diagram in the form of vector polygons. Results of the paper are developed mathematical model of automatic machines based on timing diagram and the connection between the parameters of the automatic machine timing diagram and equations of motion mechanisms through functions of position and transfer functions of mechanisms.
\end{abstract}

Index Terms-Automatic machine, dynamic, timing diagram, vector model.

\section{INTRODUCTION}

Theory of timing diagram is one of the main parts of the design theory of automatic machines. A detailed analysis of the works on the theory of timing diagram performed prior to 1965 is given in [1].

Timing diagram is a sequence of machine operations performed by mechanisms depending on the angular displacement of the main shaft. Timing diagram allows determining to the state of dwell or motion of each of the executive body at any position of the main shaft, see Fig. 1 [2].

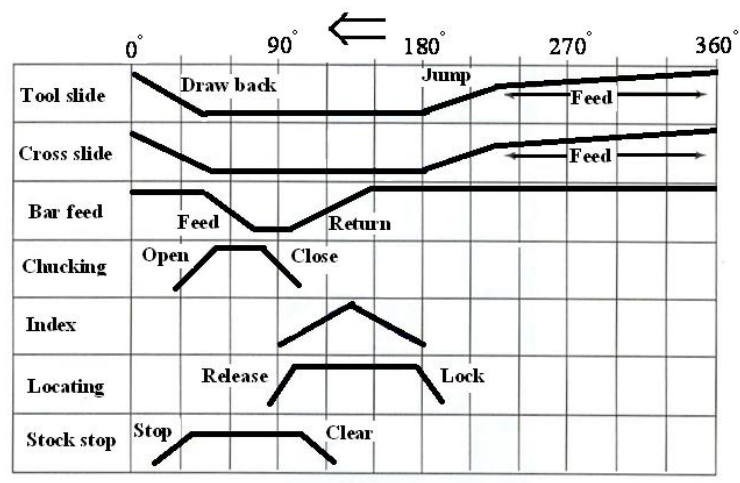

Fig. 1. Linear timing diagram of working and auxiliary cams of a four-spindle bar automatic.

Manuscript received December 11, 2013; revised February 11, 2014

The authors are with the Institute Mechanics and Mechanical Engineering, Almaty, 050010, Kazakhstan (e-mail: legsert@mail.ru, dgpimmash@mail.ru, temirbekove@mail.ru).
Analysis of the methods of synthesis and analysis of mechanisms timing diagram automatic machine [3]-[10] showed the need for further development of optimization methods timing diagram, taking into account the accuracy of manufacture and mechanisms of automatic machine connections executive for the displacement mechanisms, dynamics of automatic machine.

\section{Vector Model of Timing Diagram Automatic MACHINE}

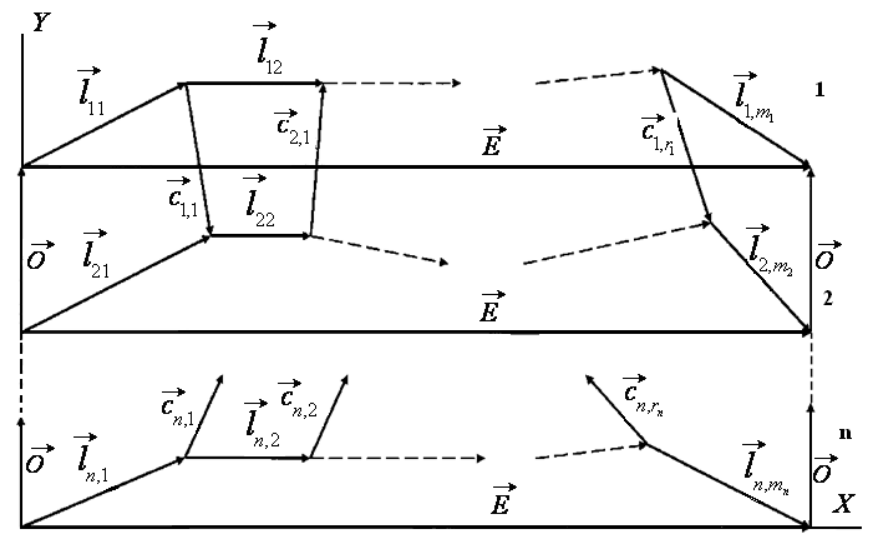

Fig. 2. Vector model of the automatic machine timing diagram

Timing diagram machines can be represented as vector polygons [11] and [12], see Fig. 2. We replace the segments linear timing diagram of the vector. Vector directed sequentially from one position to another position of mechanism. We denote the vector of the letter $\vec{\ell}_{i j}$, where $n$ - the number of mechanisms, $i$ - the number of mechanisms, $j$ - the number of position of $i$ - mechanism, $m_{j}$ - the number of positions of $i$ - mechanism.

The projection of vectors $\vec{\ell}_{i j}$ on the $\mathrm{X}$ axis is $\alpha_{i j^{-}}$phase angles actuation mechanisms. The projection $\vec{\ell}_{\ell}$ on the $\mathrm{Y}$ axis is the displacement $\delta_{i j}$ of $j$-position of $i$ - mechanism.

$$
\delta_{i j}=\frac{S_{i j}}{S_{\max }}, \quad S_{\max }=\max S_{i j}, \quad i=1, \ldots, n ; j=1, \ldots, m_{i},
$$

where $S_{i j}$ the displacement of $j$ - position of $i$ - mechanism.

We introduce the vector $\vec{E}$ connecting the point of beginning and end of the cycle. The projection of the vector $\vec{E}$ on the $\mathrm{X}$ axis is $2 \pi$ on the $\mathrm{Y}$ axis is zero. The interaction 
mechanisms with each other will reflect in the form of vectors of connection $\vec{c}_{i k}$, where $k=1, \ldots, r_{i}, r_{i}$ - the number of vectors of connection of $i$ - mechanism.

The projection of the vectors of connection to the $\mathrm{X}$-axis is the amount of delay activation mechanism, and the projection on the $\mathrm{Y}$ axis is the difference between the displacements of mechanisms.

We impose the timing diagram mechanisms at each other with zero vectors $\vec{O}$ (see Fig. 2.) connecting the boundary points timing diagram mechanisms for the $\mathrm{Y}$ axis.

Set up a system of vector equations describing the works of mechanisms automatic machine, see Fig. 2.

$$
\left.\begin{array}{l}
\sum_{j=1}^{m_{i}} \vec{\ell}_{i j}=\vec{E}, i=1, \ldots, n, \\
\vec{c}_{i k}=\sum_{i=1 j=1}^{n} \sum_{i j}^{m_{i j}} b_{i j} \vec{\ell}_{i}
\end{array}\right\}
$$

where $b_{i j} \in\{0, \pm 1\}$.

Project the vector equations (1) on the axis $\mathrm{X}$ and $\mathrm{Y}$.

$$
\left.\begin{array}{l}
\sum_{j=1}^{m} \alpha_{i j}=2 \pi, \sum_{j=1}^{m_{i}} \delta_{i j}=0, \\
c_{i k}^{x}=\sum_{i=1}^{n} \sum_{j=1}^{m_{i}} b_{i j} \alpha_{i j}, c_{i k}^{y}=\sum_{i=1}^{n} \sum_{j=1}^{m} b_{i j} \delta_{i j},
\end{array}\right\}
$$

On the phase angles of actuation of mechanisms $\alpha_{i j}$, and displacements of mechanisms $\delta_{i j}$ impose constraints

$$
\alpha_{i j} \geq \alpha_{i j}^{m}, \quad \delta_{i j}^{b} \geq \delta_{i j} \geq \delta_{i j}^{h}
$$

where $\alpha_{i j}^{m}$ - the minimum allowable phase angles of actuation of mechanisms, $\delta_{i j}^{b}, \delta_{i j}^{h}$ - the upper and lower limits assigned by the designer.

On the projection vectors of connection impose constraints

$$
c_{i k}^{x b} \geq c_{i k}^{x} \geq c_{i k}^{x h}, \quad c_{i k}^{y b} \geq c_{i k}^{y} \geq c_{i k}^{y h}
$$

where

$$
c_{i k}^{x h}=e_{i k}^{x}+\Delta c_{i k}^{x}, \quad c_{i k}^{y h}=e_{i k}^{y}+\Delta c_{i k}^{y}
$$

where $e_{i k}^{x}, e_{i k}^{y}$ - the minimum permissible projection vectors of connection, $\Delta c_{i k}^{x}, \Delta c_{i k}^{y}$ - the error of the projections of vectors of connection, $c_{i k}^{x b}, c_{i k}^{y b}$ - the upper limit imposed by the designer.

Equations (2) and constraints (3, 4) describe the collaboration works of mechanisms (timing diagram) of automatic machine.

\section{Mathematical Model of Automatic Machine BASED ON TIMING DIAGRAM}

Define the connection between the differential equations of motion of the automatic machine and the equations describing its timing diagram. Fig. 3 shows the dynamic model of the machine, where indicated $C_{i}$ - the elasticity coefficients, $\beta_{i^{-}}$ the coefficients of resistance, $J_{i}, I_{i}$ - the moments of inertia, $M_{D^{-}}$ the motor torque, $M_{i^{-}}$moment of resistance, $P_{i}, \quad i=1, \ldots, n-\mathrm{a}$ function of the mechanisms.

To compile the equations of motion mechanisms for automatic machine dynamic model, see Fig. 3, we use of Lagrange equations [13]:

$$
\left.\begin{array}{l}
\frac{d}{d t}\left(\frac{\partial T}{\partial \dot{\varphi}_{j}}\right)-\frac{\partial T}{\partial \varphi_{j}}+\frac{\partial V}{\partial \varphi_{j}}=Q_{j}+\sum_{i=1}^{m} \lambda_{i} h_{i j} \\
\sum_{j=1}^{m+n} h_{i j} \dot{\varphi}_{j}+h_{i}=0,
\end{array}\right\}
$$

where $\varphi_{1}, \varphi_{2}, \ldots, \varphi_{n}$ - the generalized coordinates, $\lambda_{i}$ - Lagrange multipliers, $h_{i j}, h_{i^{-}}$some functions, $T$ - the kinetic energy of a holonomic system, $V$ - the potential energy of the system, $Q_{j}$-the generalized force,

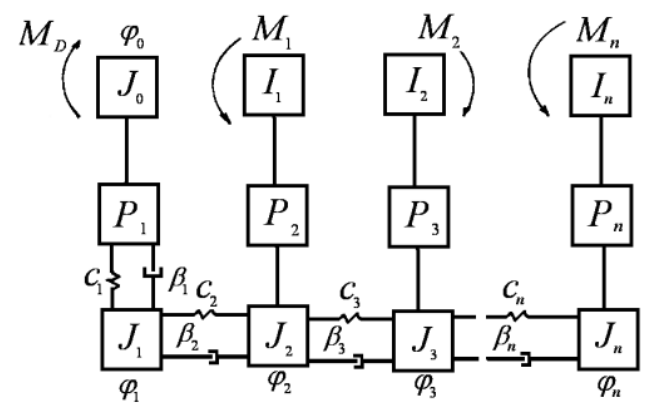

Fig. 3. A dynamic model of automatic machine.

To establish the connection between the equations timing diagram (2-4) and the dynamic equations (5), we write the functions of position, the transfer functions of mechanisms of automatic machine in the following form:

$$
\left.\begin{array}{l}
P_{i}=P_{i 1} \cdot\left[1-L\left(\varphi_{i}-\alpha_{i 1}\right)\right]+ \\
+\sum_{j=2}^{m} P_{i j}\left[1-L\left(\varphi_{i}-\sum_{r=1}^{j} \alpha_{i r}\right)\right] \cdot L\left(\varphi_{i}-\sum_{r=1}^{j-1} \alpha_{i r}\right) \\
P_{i}^{\prime}=P_{i 1}^{\prime}\left[1-L\left(\varphi_{i}-\alpha_{i 1}\right)\right]+ \\
+\sum_{j=2}^{m} P_{i j}^{\prime}\left[1-L\left(\varphi_{i}-\sum_{r=1}^{j} \alpha_{i r}\right)\right] \cdot L\left(\varphi_{i}-\sum_{r=1}^{j-1} \alpha_{i r}\right) \\
P_{i}^{\prime \prime}=P_{i 1}^{\prime \prime}\left[1-L\left(\varphi_{i}-\alpha_{i 1}\right)\right]+ \\
+\sum_{j=2}^{m} P_{i j}^{\prime \prime}\left[1-L\left(\varphi_{i}-\sum_{r=1}^{j} \alpha_{i r}\right)\right] \cdot L\left(\varphi_{i}-\sum_{r=1}^{j-1} \alpha_{i r}\right)
\end{array}\right\}
$$


where $i=1, \ldots, n, L(x)$ - a step function of the form

$$
L(x)=\left\{\begin{array}{l}
0, x<0 \\
1, x \geq 0
\end{array}\right.
$$

$P_{i j}, P_{i j}^{\prime}, P_{i j}^{\prime \prime}$ - function of position, the first transfer function, the second transfer function on parts of phase angles actuation $\alpha_{i j}$ of mechanisms

Equation (6) establish a connection between the equations (5) describe the dynamics of the automatic machine and the equations (2)-(4) timing diagram of the machine--automaton. This method allows to solve various optimization tacks, where the variable parameters are used the phase angles $\alpha_{i j}$ and of the displacements $\delta_{i j}$ of timing diagram automatic machine.

\section{EXAMPLE}

We will show in more detail the connection between timing diagram of automatic machine dynamics and mechanisms on the example of automatic machine with two cam mechanisms.

The dynamic model is shown in Fig. 4 , where $\varphi_{0}, \varphi_{1}, \varphi_{2}-$ generalized coordinates, $M_{D}$ - the motor torque, $M_{1}, M_{2}$ - the moments of resistance, $I_{1}, J_{0}, J_{1}, J_{2}$ - the moments of inertia of mechanisms, $c_{0}, c_{1}$ - the coefficients of elasticity of shafts,

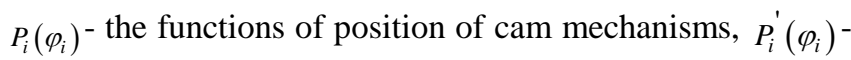
the first transfer functions of the cams, $P_{i}^{\prime \prime}\left(\varphi_{i}\right)^{-}$the second transfer functions of the cams.

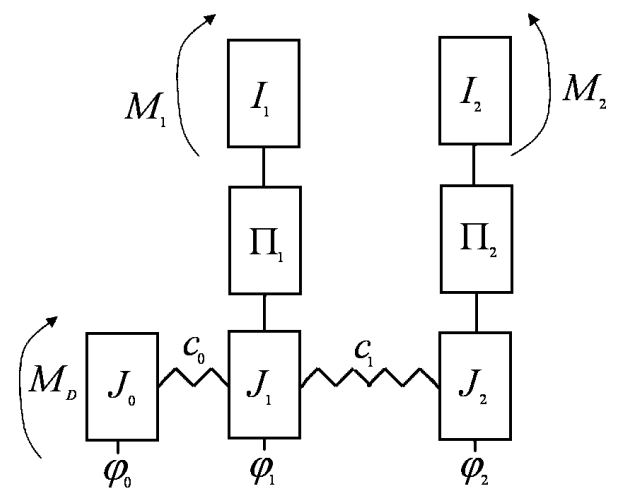

Fig. 4. Dynamic model of automatic machine with two cams mechanisms.

This dynamic model is described by the following equations

$$
\begin{aligned}
& J_{0} \ddot{\varphi}_{0}+c_{1}\left(\varphi_{0}-\varphi_{1}\right)=M_{D}, \\
& \left(J_{1}+I_{1} P_{1}^{2}\left(\varphi_{1}\right)\right) \ddot{\varphi}_{1}+I_{1} P_{1}^{\prime}\left(\varphi_{1}\right) P_{1}^{\prime \prime}\left(\varphi_{1}\right) \dot{\varphi}_{1}^{2}+ \\
& +c_{0}\left(\varphi_{1}-\varphi_{0}\right)+c_{1}\left(\varphi_{1}-\varphi_{2}\right)=-M_{1} P_{1}^{\prime}\left(\varphi_{1}\right), \\
& \left(J_{2}+I_{2} P_{2}^{\prime 2}\left(\varphi_{2}\right)\right) \ddot{\varphi}_{2}+I_{2} P_{2}^{\prime}\left(\varphi_{2}\right) P_{2}^{\prime \prime}\left(\varphi_{2}\right) \dot{\varphi}_{2}^{2}+ \\
& +c_{2}\left(\varphi_{1}-\varphi_{2}\right)=-M_{2} P_{2}^{\prime}\left(\varphi_{2}\right)
\end{aligned}
$$

where

$$
P_{i}^{\prime}\left(\varphi_{i}\right)=\frac{d P_{i}\left(\varphi_{i}\right)}{d \varphi_{i}} ; P_{i}^{\prime \prime}\left(\varphi_{i}\right)=\frac{d^{2} P_{i}\left(\varphi_{i}\right)}{d \varphi_{i}^{2}} ; i=1,2
$$

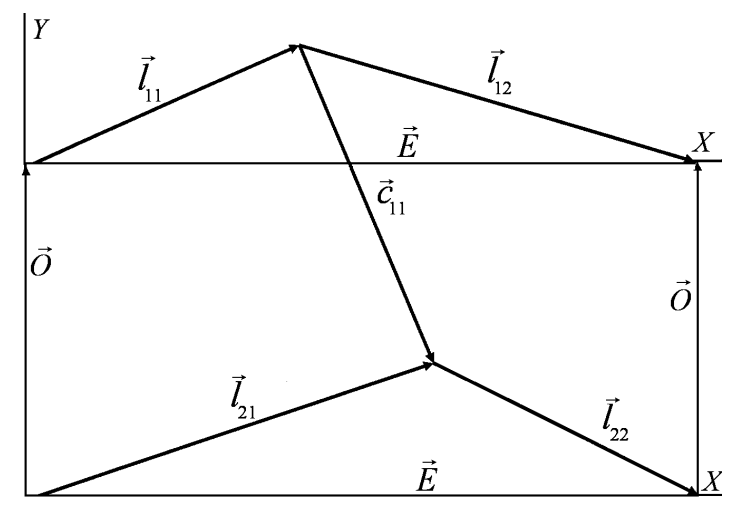

Fig. 5. Vector timing diagram of automatic machine with two cams mechanisms.

Fig. 5 shows vector timing diagram of automatic machine, which is described by the following equations:

$$
\left.\begin{array}{l}
\vec{l}_{11}+\vec{l}_{12}=\vec{E} \\
\vec{l}_{21}+\vec{l}_{22}=\vec{E} \\
\vec{c}_{21}=\vec{l}_{21}-\vec{l}_{11}
\end{array}\right\}
$$

We project (8) on the $x, y$

$$
\left.\begin{array}{c}
\alpha_{11}+\alpha_{12}=2 \pi \\
\alpha_{21}+\alpha_{22}=2 \pi \\
c_{11}^{x}=\alpha_{21}-\alpha_{11}
\end{array}\right\}
$$

Impose constraints on the phase angles, displacements of mechanisms, and projections of vectors of connection

$$
\left.\begin{array}{l}
\alpha_{i j} \geq \alpha_{i j}^{\min } \\
\delta_{i j}^{\max } \geq \delta_{i j} \geq \delta_{i j}^{\min } \\
c_{11}^{x \max } \geq c_{11}^{x} \geq c_{11}^{x \min } \\
c_{11}^{y \max } \geq c_{11}^{y} \geq c_{11}^{y \min }
\end{array}\right\}
$$

The expressions (9-11) allow you to vary the phase angles and displacements of mechanisms of automatic machine, without disrupting their normal work.

To establish the connection between the equations describing of joint work of the mechanisms of automatic machine (9-11) and the dynamic equations (7), we write the functions of position and the transfer functions of mechanisms of automatic machine, see Fig. 6, as follows: 


$$
\left.\begin{array}{l}
P_{i}=P_{i 1} \cdot\left[1-L\left(\varphi_{i}-\alpha_{i 1}\right)\right]+ \\
+P_{i 2}\left[1-L\left(\varphi_{i}-\left(\alpha_{i 1}+\alpha_{i 2}\right)\right)\right] \cdot L\left(\varphi_{i}-\alpha_{i 1}\right) \\
P_{i}^{\prime}=P_{i 1}^{\prime} \cdot\left[1-L\left(\varphi_{i}-\alpha_{i 1}\right)\right]+ \\
+P_{i 2}^{\prime}\left[1-L\left(\varphi_{i}-\left(\alpha_{i 1}+\alpha_{i 2}\right)\right)\right] \cdot L\left(\varphi_{i}-\alpha_{i 1}\right) \\
P_{i}^{\prime \prime}=P_{i 1}^{\prime \prime} \cdot\left[1-L\left(\varphi_{i}-\alpha_{i 1}\right)\right]+ \\
+P_{i 2}^{\prime \prime}\left[1-L\left(\varphi_{i}-\left(\alpha_{i 1}+\alpha_{i 2}\right)\right)\right] \cdot L\left(\varphi_{i}-\alpha_{i 1}\right), i=1,2
\end{array}\right\}
$$

where

$$
L(x)=\left\{\begin{array}{l}
0, x<0 \\
1, x \geq 0
\end{array}\right.
$$

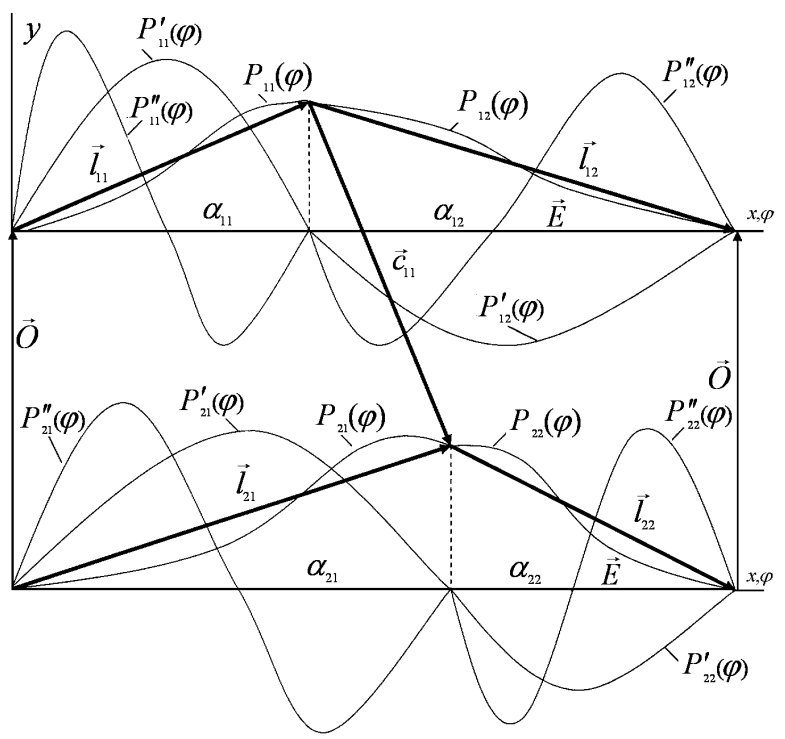

Fig. 6. The functions of position and the transfer functions of mechanisms of automatic machine.

Represent the generalized coordinates $\varphi_{1}, \varphi_{2}$ through the dimensionless coefficients $k_{1}, k_{2}$, where

$$
\begin{gathered}
\varphi_{1}=2 \pi k_{1} ; \varphi_{2}=2 \pi k_{2} ; k_{1}, k_{2} \in[0,1] \\
P_{i j}=a_{i j}\left(k_{i}\right) \delta_{i j}, P_{i j}^{\prime}=b_{i j}\left(k_{i}\right) \frac{\delta_{i j}}{\alpha_{i j}}, \\
P_{i j}^{\prime \prime}=d_{i j}\left(k_{i}\right) \frac{\delta_{i j}}{\alpha_{i j}^{2}}, i=1,2 ; \mathrm{j}=1,2 ; \\
a_{i j}\left(k_{i}\right), b_{i j}\left(k_{i}\right), d_{i j}\left(k_{i}\right) ; i=1,2 ; j=1,2 ;
\end{gathered}
$$

Coefficients of the displacement, the velocity, the acceleration of mechanism in $j$ - position.

Equations (12), (13) establish a connection between the phase angles of actuation of mechanisms $\alpha_{i j}$ and magnitude of displacements of mechanisms $\delta_{i j}$ of their functions of position and transfer functions that are explicitly included in the equations of motion of the automatic machine (7). Now, depending on the chosen optimization criterion, by varying the parameters of timing diagram $\alpha_{i j}$ and $\delta_{i j}$, can improve the dynamics of the automatic machine.

\section{CONCLUSIONS}

The vector model timing diagram is developed on the basis of representation timing diagram of the automatic machine as vector polygons, which allows solving various dynamic tasks at the expense of change of parameters timing diagram. The mathematical model of the automatic machine with elastic links and with the account timing diagram of its mechanisms is received. The equations of connection between parameters timing diagram of the automatic machine and equations of dynamics through functions of position and transfer functions of mechanisms are received.

\section{REFERENCES}

[1] L. V. Petrokas, "Reviews of timing diagram manufacturing machines and automatic production lines," J. Theory of automatic machines and pneumatic, vol. 1, pp. 22-36, May 1970.

[2] J. W. Browne, The Theory of Machine Tools, Cassell and Co. Ltd. 1965 , pp. 365

[3] V. A. Novgorodtsev, "Presentation of the machine timing diagram as a graph," Theory of Mechanisms and Machines, vol. 33, pp. 57-60, July 1982.

[4] D. Homer, Kinematic Design of Machines and Mechanisms, McGraw-Hill, New York, 1998, pp. 661.

[5] B. Z. Sandler, Robotics: Designing the Mechanisms for Automated Machinery, Academic Press, 1999, pp. 433.

[6] C. Natale, Interaction Control of Robot Manipulators: Six Degrees-of-Freedom Tasks, Springer-Verland, Berlin, 2003, pp. 108.

[7] S. K. Bhattacharya and B. Singh, Control of Machines, New Age International, New Delhi, 2006, p. 355.

[8] N. Board, Complete Technology Book on Textile, Spinning, Weaving, Finishing and Printing, Asia Pacific Business press Inc., 2009, pp. 814.

[9] L. R. Norton, Cam Design and Manufacturing Handbook, Industrial Press Inc., 2009, pp. 591.

[10] M. Topalbekiroglu and H. I. Celik, "Kinematic analysis of beat-up mechanism used for handmade carpet looms," Indian Journal of Fibre \& Textile Research, vol. 34, pp. 129-136, Jan. 2009.

[11] A. Jomartov, "Dynamics of machine-automaton jointly with cyclogram," in Proc. World Congress on Engineering, London, 2010, pp. 1224-1229.

[12] A. Jomartov, "Multi-objective optimization of cyclogram mechanisms machine-automaton," in Proc. World Congress on Engineering, London, 2011, pp. 2562-2565.

[13] I. I. Wolfson, Dynamics Calculations of Cycle Mechanisms, Leningrad, 1976, p. 386.

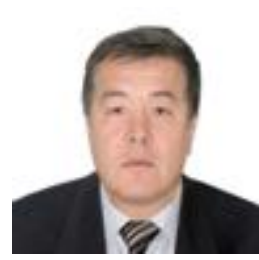

Assylbek Jomartov was born on July 28, 1961 in Almaty. He is a doctor of technical sciences, professor.

$\mathrm{He}$ is the vice-general director of Institute of Mechanics and Mechanical Engineering. His research interests cover of the dynamics of automatic machines with elastic links with the timing diagram for the development of mechanisms for the dynamic analysis of automatic machines. He is the author or co-author of scientific work of more than one hundreds, which have been presented at conferences or published in national and international journals and 6 patents.

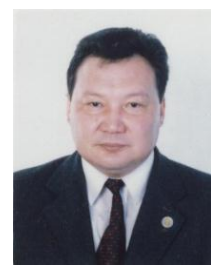

Skanderbek Joldasbekov was born on March 9, 1955 in Almaty. He is a doctor of technical sciences, professor. He is the general director of Institute of Mechanics and Mechanical Engineering. His research interests cover of the analysis and synthesis of the linkage mechanisms of high classes. He is author or co-author of scientific work of more than175, which have been presented at conferences or published in national and international journals and 30. patents.

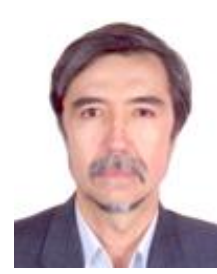

Yerbol Temirbekov was on born March 9, 1955 in Almaty. He is a doctor of technical sciences, professor $\mathrm{He}$ is a professor of Institute of Mechanics and Mechanical Engineering. His research interests cover of the dynamics of the pivotal mechanisms. He is the author or co-author of scientific work of more than one hundreds, which have been presented at conferences or published in national and international journals and 10 